\title{
Problématique du genre dans les situations de détresse : le cas des réfugiés en Afrique
}

\author{
Danièl e Lal iber té-Beringar
}

\section{Résumé}

Le présent article vise à faire le point sur les rapports hommes/femmes dans les zones de regroupements liées aux guerres et surtout dans les camps de réfugiés. II vise également à montrer comment les situations de conflit accentuent les inégalités entre les sexes à tous les âges. Pour ce faire, nous avons consi déré la littérature scientifique de la dernière décennie, mais également les politiques et programmes du $\mathrm{H}$ aut Commissariat des $\mathrm{N}$ ations $\mathrm{U}$ nies pour les réfugiés (HCR). Le texte est divisé en sept composantes principales : la place du genre dans la recherche sur les réfugiés, dansl'aide internationale aux réfugiés et dans le droit des réfugiés; un survol statistique, la transformation des rôles associés au genre, l'impact différentiel du déplacement selon le genre, la vulnérabilité des femmes et des filles réfugiées.

\section{Abstract}

This article tries to take stock of male/female relationships in assembly areas related to wars, and especially in refugee camps. It also aims at showing how conflicts heighten inequalities between the sexes at every age level. The approach adopted has been to review scientific literature of the last decade, as well as the policies and programs of the UN H igh Commissioner for Refugees (UN HCR). The text is divided into several main parts: the place occupied by gender in research on refugees, in international aid, and refugee rights; a statistical overview; the transformation of the roles associated with gender; the difference in the impact of displacement depending on gender; and the vulnerability of refugee women and girls.

\section{La plac} place du genre dans la recherche sur les réfugiés es études sur les réfugiés, ainsi que les études féministes du développement et de la migration forcée, ne se sont que récemment intéressées à la problématique du genre dans le cas des réfugiés. Nombreux sont les chercheurs et les praticiens - en majorité des hommesoccidentaux - qui auparavant traitaient les réfugiés comme un groupe homogène inconsciemment assimilé à l'image de l'adulte mâle.

II est possible de tracer un parallèle entre l'évolution de la théorie féministe, de la recherche sur la migration forcée et de la prise en compte du genre dans le développement ${ }^{1}$. L'approche centrée sur la femme dans la migration forcée a été adoptée (Women in Forced Migration ou WIFM) parallèlement à celle dans le développement (Women in Development ou WID). Par la suite, l'approche fondée sur legenreet la migration forcée(Gender and Forced M igration ou GAFM ) a été élaborée conjointement avec l'approche genre et développement (Gender and Development ou GAD). D ans les faits, I'approche femme dans la migration forcée est toujours prépondérante et renforce la marginalisation del'expérience féminine ${ }^{2}$. En effet, les analyses considèrent le traitement différentiel accordé aux femmes en raison de leur sexe, plutôt que de se concentrer sur la construction de l'identité de genre sel on le contexte géographique, historique, politique et socio-culturel.

II y a eu cependant un accroissement des recherches sur lesfemmes réfugiées durant les annéesquatre-vingt. T outefois, seules des recherches marginales portant sur des questions typiquement féminines se sont penchées sur les femmes réfugiées ou les structures degen $\mathrm{e}^{3}$. Ces recherches sont demeurées exploratoires et n'ont pas mis en évidence les différences basées sur le sexe4. On a négl igé l'impact du statut de réfugié sur la vie individuelle et sur la relation des femmes avec les hommes 5 . De plus, lorsqu'il est pris en compte, le genre n'est qu'une variable parmi d'autres. L'augmentation de l'usage du mot genre dans le contexte 
des migrations forcées n'implique pas nécessairement que les programmes soient davantage sensibilisés au genre en cause. Enfin, l'application de l'approche en fonction du genre dans la migration forcée requiert de profonds changements institutionnels.

\section{La place du genre dans l'aide internationale aux réfugiés}

Les relations de genre structurent la vie des réfugiés et l'administration de l'assistance humanitaire ${ }^{6}$. Ce n'est que depuis l'exode des réfugiés indochinois en 1978-81 que les organismes d'aidehumanitaire ont commencéà prendre en compte l'effet du genre sur l'expérience de refuge, dans la formulation de leurs politiques et la planification de leurs interventions. Un certain nombre d'événements internationaux ont accéléré le processus en amenant de multiples intervenants à partager leurs expériences et à développer des stratégies communes. Ainsi, en 1981, l'Intergovernmental Committeefor $M$ igration a organisé un séminaire sur l'adaptation et l'intégration des femmes réfugiées et migrantes. En 1985, le HCR a organisé une table ronde sur la femme réfugiée à laquelle ont participé des politiciens et des organismes non gouvernementaux (ONG). La première recommandation du HCR portant sur la protection de la femme réfugiée a d'ailleurs été adoptée par le comité de direction cette annéelà. En 1987, le comité de direction du HCR a admis que des directives devaient être établies afin d'améliorer l'assistance et la protection de la femme réfugiée. C'est ainsi qu'il a mis en place un groupe de travail chargé de revoir ses programmes. Puis, en novembre 1988 a eu lieu la première consultation sur la femme réfugiée, organi sée par le N GO Working Group on Refugee Women, à laquelle ont participé des groupes de réfugiés, des organismes internationaux et des institutions d'enseignement. II en a résulté un guide visant à répondre aux besoins spécifiques des femmes réfugiées.

Durant la dernière décennie, en s'inspirant du plan d'action de Beijing, la politique et les activités du HCR ont évolué en visant l'égalité entre les genres? ${ }^{7}$. Ainsi, le HCR a publié en 1990 une politique sur la femme réfugiée visant à faciliter l'accès et la participation des femmes à ses programmes ${ }^{8}$. Puis, en 1991, il a produit un guide sur la protection de la femme réfugiée. Ce guide vise à améliorer la situation des femmes et à répondre à leurs besoins spécifiques, et ceen considérant leursrôlesproductifs et sociaux. Un programme de formation du personnel (People-Oriented Planning ou POP) a vu lejour en 1992, afin de mieux identifier et adresser les besoins des réfugiés selon leur genre. Ce programme (révisé en 1999) est devenu le principal outil facilitant l'application de la politique sur la femme réfugiée. II est basé sur le principe quel'analyse des rôles sociaux et économiques joués par les femmes, les hommes et les enfants dans la communauté est un pré requis à la planification des activités de protection et d'assistance des réfugiés 9 . LeHCR a ensuite produit un document portant sur la violence sexuelle à l'égard des réfugiées ${ }^{10}$.

Cependant, une évaluation interne ${ }^{11}$ aurait démontré que la politique du HCR n'aura d'impact que si ce dernier effectue des changements institutionnels ${ }^{12}$. Bien quele progrès fait par le HCR face à l'application de sa politique en Afrique soit variable, le rôle des femmes africaines dans le développement des programmes pour les réfugiés et dans l'administration des camps et des établissements devient prépondérant ${ }^{13}$. Lors de la revue (juin 2000) des progrès accomplis par la communauté internationale depuis Beijing, on a reconnu les efforts faits en vue de protéger les droits des femmes et des filles dans des situations de conflit $^{14}$. Cependant, on recommande de nouvelles actions nationales et internationales afin d'assurer la participation réelle des femmes dans les décisions et la mise en place d'activités de développement et de processus de paix. La conférence internationale sur les enfants affectés par la guerre, tenue à Halifax en septembre 2000, a réitéré la nécessité de mieux tenir compte du genre dans les missions de maintien et de consolidation de la paix ${ }^{15}$.

\section{Le genre dans le droit des réfugiés}

Les instruments juridiques internationaux permettant de gérer l'assistance et la protection des réfugiés depuis les années cinquante (tels quela Convention des $\mathrm{N}$ ations U nies de 1951, le Protocole de 1967 et la Convention de I'OUA de 1969) ${ }^{16}$ sont basés sur le postulat que les femmes et les hommes vivent les situations de crise de la même manière. La principale critique féministe du concept de réfugié proposé par ces conventions est qu'elles ne reconnaissent que les formes de persécution frappant les hommes. Elles ne mentionnent d'aill eurs pas explicitement l'oppression basée sur le genre ${ }^{17}$.

Comme les femmes sont plus rarement actives dans la sphère publique, elles participent peu aux groupes politiques, religieux ou raciaux susceptibles d'être persécutés. Par ailleurs, la violence contre les opposants à un régime n'est pas confinée à la sphère publique, elle se manifeste parfois sous la forme de violence dans le ménage et la famill $\mathrm{e}^{18}$. Les femmes sont discriminées sur la base de leur sexe et de la position sociale subordonnée de leur genre, victimes d'actes commis à des fins d'intimidation, de gratification sexuelle ou d'humiliation. Elles sont parfois torturées ou persécutées en raison des activités politiques d'un membre de leur famille. Elles sont égal ement discriminées pour avoir transgressé les lois ou les normes sociales ${ }^{19}$. L'essor du fondamentalisme islamique favorise la discrimi- 
nation et la persécution des femmes qui refusent d'adhérer aux codes de conduite prescrits par la religion ${ }^{20}$. Cependant, les formes de violence(dont leviol) contreles femmes ne sont pas vues comme des violations des droits humains en vertu desquelles elles pourraient prétendre être persécutées ${ }^{21}$.

C'est durant la Décennie de la femme (1976-1985), proclamée par I'Assemblée générale des Nations U nies, que la communautéinternationalea manifestéun intérêt grandissant à l'égard des problèmes et des besoins spécifiquement féminins ${ }^{22}$. Bien quela persécution baséesur legenrene soit toujours pas mentionnée dans les instruments juridiques internationaux, certains juristes ont reconnu à partir des années quatre-vingt la nécessité de réinterpréter la notion de persécution pour permettre aux femmes de réclamer le statut deréfugiée. LeH CR a donc clarifié son interprétation de la définition : les femmes persécutées en raison de leur appartenance à un groupe social particulier (le groupe des femmes) peuvent maintenant prétendre au statut légal de réfugiée. II encourage les gouvernements à mettre en place des procédures d'asile prenant en compteles questions relatives au genre. C'est ainsi que le Canada (1993), les États-Unis (1995), l'Australie (1996) et le Royaume-Uni (2000) ont développé des directives spécifiquement pour les demandes d'asile des femmes ${ }^{23}$. Le HCR tente maintenant de favoriser l'égalité de genre dans ses programmes et de recruter du personnel féminin pour traiter les requêtes de statut ${ }^{24}$.

\section{Un survol statistique}

On mentionne généralement dans la littérature que les femmes et les enfants dépendants forment de $75 \%$ à $80 \%$ des réfugiés et des personnes déplacées. Cette proportion atteindrait d'ailleurs plus de $90 \%$ chez certaines populations réfugiées ${ }^{25}$. Les lacunes des données empiriques ont pour conséquence des généralisations appliquées à tort à toutes les situations ${ }^{26}$. Or, selon les statistiques du HCR pour l'année 1999 (tableau 1), les femmes ne constituent que 49,7\% des 2341850 personnes relevant de son mandat et pour lesquellesil connaît la structure démographique dela population. Par ailleurs, les femmes sont majoritaires en Afrique de l'Ouest $(52,8 \%)$ et en Afrique du Nord $(50,9 \%)$. La distribution par âge indique que dans toutes les régions, le pourcentage de femmes est généralement plus élevé que celui des hommes chez les soixante ans et plus ( $52,7 \%$ pour I'ensemble de l'Afrique). Ce pourcentage est toutefois inférieur pour les autres groupes d'âge au sud del'Afrique, en Afrique centraleet de l'Est. Par contre, en Afrique de l'O uest il est supérieur dans tous les groupes d'âge, tandis qu'en Afrique du Nord il n'est inférieur que chez les 5-7 ans. Cependant, ces données ne reflètent que partiellement la situation, puisque le HCR ne connaît pas la structure démographique de l'ensemble des réfugiés, et parce qu'il se concentre plus particulièrement sur les personnes dont il est responsable.

II est donc nécessaire de considérer chaque situation dans sa spécificité. Ainsi, deux recensements des réfugiés

\begin{tabular}{|l|c|c|c|c|c|c|c||}
\hline \multicolumn{8}{|c|}{ Tableau 1 } \\
Pourcentage de femmes par groups d'åge et région d'asile, Afrique, fin 1999 \\
\hline Région d'asile & $0-4$ & $5-17$ & $18-59$ & $60+>$ & $\begin{array}{c}\text { Autre } \\
\text { inconnus }\end{array}$ & Total & $\begin{array}{c}\text { Nombre } \\
\text { (sexes réunis) }\end{array}$ \\
\hline \hline Afrique centrale & 45,5 & 45,6 & 47,6 & 52,0 & 51,4 & 48,2 & 166260 \\
\hline Afrique de l'Est & 49,9 & 47,9 & 46,6 & 50,8 & 50,1 & 48,1 & 1159660 \\
\hline Afrique du Nord & 51,7 & 48,0 & 53,1 & 56,7 & $\ldots$ & 50,9 & 168040 \\
\hline Afrique de l'Ouest & 51,6 & 50,8 & 54,8 & 53,6 & 60,1 & 52,8 & 815280 \\
\hline Sud de l'Afrique & 49,7 & 44,0 & 28,8 & 51,9 & $\ldots$ & 33,3 & 32610 \\
\hline \hline Total Afrique & 50,5 & 48,9 & 49,5 & 52,7 & 51,0 & 49,7 & 2341850 \\
\hline \hline
\end{tabular}

Source: UNHCR (2000c). U NH CR Global Report 1999.

Note: la composition démographique est disponible pour un ensemble de 5,4 millions de personnes I'Note : la composition démographique est disponible pour un ensemble de 5,4 millions de personnes sur l'ensemble des continents. Les données fournies dans ce tableau nereprésentent donc pas nécessairement le nombre total de réfugiés dans chaque pays. Ainsi, au premier janvier 2000, plus de 6250540 individus relevaient du mandat du HCR en Afrique. 
(1976 et 1988) et une enquête (1986-1987) réalisés dans un établissement de réfugiés burundaisen Tanzanie ont démontré que les femmes ne sont pas plus nombreuses. Dans les camps de réfugiés somaliens au Kenya (H agadera), on a observé un surplus de $10 \%$ de femmes dans les groupes d'âge allant de 19 à 44 ans, maisuneinsuffisance defemmesau-dessus de 45 ans $^{27}$. Le surplus de femmes serait dû à l'effet de la migration sélective, les hommes s'absentant du camp pour exercer des activités professionnelles ou militaires. Les vieilles femmes resteraient dans le pays d'origine plus fréquemment quelesvieux, ou aurai ent un taux demortalitéplusélevé. Dans les camps de réfugiés ougandais au Soudan (1982), certaines femmes ont été désignées à tort chefs de ménage parce que leur mari était associéau ménage d'une coépouse2².

Une étuderéal isée auprès des réfugiés éthiopiensen 1988 a par ailleurs démontré que les ménages dirigés par des femmes ne sont pas plus nombreux queceux dirigés par des hommes ${ }^{29}$. U ne autre étude auprès des réfugiés éthiopiens au Soudan a quant à elle révélé que les hommes sont majoritaires dans cinq des six établissements étudiés ${ }^{30}$. Le fait remarquable est queles ménages dirigés par des femmes sont plus nombreux qu'ils ne devraient l'être dans une population patrilinéai re et patrilocale. En effet, près de $15 \%$ des ménages sont dirigés par une femme, le phénomène étant deux fois plus fréquent dans les sites organisés que dans les établissements spontanés. D'autres recherches, toutefois limitées aux sites organisés, démontrent que de $20 \%$ à $30 \%$ des ménages réfugiés au Soudan sont dirigés par une femme. Les exigences de la fuite et du processus de déracinement seraient à l'origine de cette situation.

Les stratégies d'assistance et de protection ne sont efficaces que lorsqu'elles tiennent compte des différences dues au genre, à la classe, à l'âge, à l'éducation, à la taille et à la composition du ménage. Dans touslescas, il est indéniable que les bouleversements survenus avant, pendant et après la fuite altèrent la structure familiale traditionnelle ${ }^{31}$. Les hommes adultes sont fréquemment tués, incarcérés ou recrutés pour des activités militaires, tandis qu'un certain nombreeffectuent des migrationsprofessionnelles. Ces migrations brisent les familles nucléaires et augmentent dramatiquement le nombre de femmes effectivement en charge des enfants et des personnes âgées. Ce phénomène a été suffisamment documenté pour affirmer qu'il est réel, malgré l'absence de statistiques ${ }^{32}$. La situation de ces réfugiées souvent devenues chef de ménage pour la première fois, est aggravée par le fait que le statut de la femme est désavantageux ${ }^{33}$. D'ailleurs, les ménages dirigés par une femme sont plus à risque. La modification de la structure démographique dela population dans les camps de réfugiés affecte le rôle et la position dévolus à chaque genre dans la communautés4.

\section{La transformation des rôles associés au genre}

Les conflits armés et les migrations forcées bouleversent les cycles de production et de reproduction en changeant la définition des rôles et les relations de genre au niveau du ménage et de la société. Dans certains cas ( par exemple les réfugiés burundais en Tanzanie), la continuité dans les relations sociales permises par la présence de parents dans le pays d'accueil perpétueet mêmerenforceles rôlesculturellement définis ${ }^{35}$.

Cependant, la crise entraînée par le statut de réfugié provoque souvent une redéfinition des rôles et comportements traditionnels associés au genre. Les bases socio-culturelles de respect entre les genres sont modifiées. L'interdépendance des identités et des rôles associés au genre peut s'accentuer, par exemple chez les réfugiés éthiopiens et éryth réens dont l'expérience du refuge a varié selon le sexe ${ }^{36}$. Les relations sociales de ces derniers ont été fragmentées, tandis que sont apparus des sentiments de contradiction, de conflit et d'ambivalence par rapport au sens de soi. Les relations entre les genres sont parfois perturbées parce que les hommes vivent une crise d'identité et subissent le déclin de leur statut socio-économique. En interrogeant les réfugiés burundais à Lukole en Tanzanie, Turner ${ }^{37}$ note que les hommes et les femmes considèrent que leur arrivée dans le camp a été suivie d'une décadence morale (prostitution, divorce, polygamie, jeunes hommes mariant des femmes plus âgés...). Alors que le HCR souhaite développer l'idéal d'égalité entre les genres, les hommes croient que l'organisme prend leur place. Son autorité sur les femmes transcende leur autorité traditionnelle, parce qu'il les a remplacés comme pourvoyeur des besoins du ménage. Paradoxalement, la politique participative du HCR afavorisél'ascension socialed'un groupe de jeunes hommes semi-éduqués tentant de regagner leur masculinité perdue.

Les programmes d'assistance jouent un rôle crucial dans la position des genres dans le nouveau contexte social. Par leur attitude, les organisations internationales, les gouvernements d'accueil et le personnel des camps de réfugiés confinent les femmes dans des rôles de production et de reproduction spécifiques ${ }^{38}$. De plus, la participation féminine au leadership communautaire est limitée.

La participation des femmes au leadership

L'axiome selon lequel les réfugiés doivent participer à la gestion de leur situation est accepté, mais son corollairen'a pas encore étéreconnu - lesfemmes devraient êtrereprésentées au même titre que les hommes ${ }^{39}$. On sous-estime la capacité d'adaptation des femmes, que l'on incite à jouer le rôlepassif devictimes ${ }^{40}$. Elles sont souvent exclues des structurespolitiques ${ }^{41}$, des débats et prises dedécision concernant 
leur avenir et celui de leur communauté42. C'est d'autant plus vrai que les camps sont planifiés par des hommes et pour des hommes, ce qui accentue la marginalisation économique et sociale des femmes ${ }^{43}$.

Selon le HCR, les femmes n'ont jamais été délibérément marginalisées, maisil reconnaît avoir insuffisamment considéré la spécificité de leur vie quotidienne parce que les porte-parole des comités de réfugiés sont général ement des hommes ${ }^{44}$. L'éducation et les capacités linguistiques des hommesfacilitent leur recrutement préférentiel pour offrir les formations et les services dans les camps de réfugiés ${ }^{45}$.

Les normes culturelles, le manque de qual ification ou un problème d'estime de soi écartent parfois les femmes de la planification et de la réalisation des programmes d'assistance. Des relations de pouvoir et une division du travail inégalitaires entre les genres existent souvent dès le milieu d'origine ${ }^{46}$, mais le déplacement amplifie ces inégalités. Le $\mathrm{HCR}$ tente de redresser ces déséquilibres en offrant des programmes de formation pour amener les femmes à contribuer effectivement à la gestion communautair $\mathrm{e}^{47}$. C ependant, l'accès aux ressources dans les camps de réfugiés demeure limité pour les femmes.

\section{L'accès aux ressources dans les camps de réfugiés}

Les administrateurs du camp, les donateurs et les membres du gouvernement sont essentiellement des hommes qui allouent les ressources aux hommes. Dans bien des sites organisés, l'accès aux ressources (terre, cultures, nourriture et habillement) est généralement contrôlé par le représentant mâle du ménage ${ }^{48}$. Le HCR remet généralement les cartes d'identification des réfugiés aux chefs de ménage, ce qui augmente le pouvoir des hommes et entraîne des difficultés économiques en cas de rupture ou quand ils abandonnent leur épouse ${ }^{49}$.

Commeles ménages dirigés par une femme comportent généralement un moindre nombre d'adultes, ils sont désavantagés par un accès différentiel au marché du travail. De plus, l'idéologie des donateurs à l'égard des rôles dévolus aux genres affecte l'offre d'activités génératrices de revenu dans les camps ${ }^{50}$. Ainsi, l'accès des femmes à l'équipement est réduit, tandis que la val eur marchande de leurs biens de production est inférieure à celle des hommes.

On a observé que les tendances patriarcales de la société d'origine et des programmes d'établissement ont défini les modalités d'accès aux ressources des réfugiés burundais en Tanzanie $e^{51}$. Comme les Burundaises ne contrôlaient pas les moyens deproduction, leur accès aux ressources leur permettait tout juste d'atteindre le seuil de subsistance. Le taux de mortalité des familles nucléaires sans homme adulte était au-dessus de la moyenne. Les relations de genre sont donc déterminantesdansla reconstruction etlasurviedesménages.
La redistribution des tâches entre les genres est souvent inévitable.

La redistribution des tâches au sein des ménages En général, la charge detravail des femmes en exil augmente, car en plus deleurs fonctions traditionnelles, elles assument certains rôles et responsabilités des hommes. M aison assiste parfois àuneredistribution destâches au sein desménages ${ }^{52}$. Ainsi, à I kafe, en Ouganda, les réfugiés soudanais assument des tâches typiquement féminines (récolte, ramassage du bois, puisage del'eau), pour éviter que leur conjointe ne se fasse violer dans les champs ou sur les routes. Les femmes seules demeurent vulnérables puisqu'elles n'ont pas accès à un tel partage de tâches.

Afin de faire vivre leur famille, elles acceptent plus facilement que les hommes d'occuper des postes très mal payés. Elles prennent alors en charge les hommes sans emploi. La contrepartie est que certains hommes, dévalorisés par leur perte de statut économique, sombrent dans l'alcoolisme et deviennent violents à leur égard. En l'absence d'occasions d'emploi, ellesdéveloppent desidéescréativesafin desurvivre. Afin d'assurer la surviedeleur famille, lesfemmestchadiennes sesont ainsi engagées dans demultiplesactivitéséconomiques dans les camps et parmi les populations locales ${ }^{53}$.

Cependant, on a observé que les changements dans les rôles modifient lecontrôle dela nourriture dans leménage. Ainsi, dès que les récoltes viennent, les hommes écartent les femmes des décisions concernant la vente, le stockage et la consommation des semences et de la nourriture ${ }^{54}$. Le système de distribution del'aide alimentaire est politisésur la base du genre ${ }^{55}$. La distribution des vivres est l'objet de rapports de force qui désavantagent les femmes et en conséquence les ménages dirigés par une femme $\mathrm{e}^{56}$. Pourtant, les femmes ont démontré leur plus grande efficacité, et ce, d'autant plus dans des situations de malnutrition, par exemple au Rwanda, où elles répondaient mieux aux besoins des démunis ${ }^{57}$.

\section{L'impact différentiel du déplacement selon le genre}

Les problèmes des femmes sont généralement abordés en référence aux droits humains universels, ou selon une étroite conception de la discrimination sexuelle face à leur accès à ces droits ${ }^{58}$. Pourtant, les femmes réfugiées subissent l'effet de la migration forcée d'une manière particulière et leurs besoins spécifiques sont souvent ignorés par les chercheurs et les praticiens. Lesconditions économiques, sociales et politiques de l'expérience passée et présente des femmes réfugiées jouent un rôle déterminant ${ }^{59}$.

Les opérations d'urgence se sont toutefois améliorées depuisquelepersonnel du HCR a reçu uneformation pour 
identifier et répondre aux besoins spécifiques des femmes et des enfants ${ }^{60}$. Le HCR a reconnu leurs besoins spéciaux en soins de santé, en équipement, en logement (par exemple, la construction de latrines éloignées de celles des hommes), ainsi que par rapport à la violence et à l'exploitation sexuelles ${ }^{61}$. Cependant, on considère d'abord les femmes comme reproductrices, tandis que leur rôle de productrices est ignoré. Les prochaines sections se rapportent au besoin fondamental que constitue la santé.

La santé différentielle des réfugiés sel on le sexe Les agences d'aide humanitaire et les chercheurs reconnaissent que legenre est un facteur déterminant de la santédans les situations de refuge. La morbidité différentiellen'est que partiellement d'origine biologique, car elle dépend aussi de la position sociale réciproque des genres, de leur perception dela maladieet del'adéquation des services médicaux offerts selon le sexe ${ }^{62}$. L'établissement et la gestion d'un camp de réfugiés doivent tenir compte des besoins pratiques des femmes enceintes et de celles ayant de jeunes enfants. Les maternités fréquentes et à risque ont d'autant plus d'impact dans les situations extrêmes où les femmes ont un accès limitéà un logement, à des conditions hygiéniques et à une alimentation convenables. C'est pourquoi dans la plupart des programmes du HCR les femmes enceintes et qui allaitent reçoivent un surplus de nourriture ${ }^{63}$.

Une recherche effectuée auprès des réfugiéssomaliensau Kenya (entre 1992 et 1996) a démontré que le contexte social particulier au camp de réfugiés affecte plus la santé des femmes, particulièrement en cas de grossesse ou d'allaitement ${ }^{64}$. Le risque vécu par celles-ci est relié à leur position sociale et à leur statut dans la société, qui limitent leur accès à la nourriture et aux autres ressources. D'une part, on soupçonne que l'allocation de la nourriture dans les ménages favorise les garçons et les hommes. D'autre part, pour assister leur mère dans les tâches domestiques, les filles se retirent plus fréquemment des programmes nutritionnels s'adressant aux jeunes. Par ailleurs, les programmes impliquent rarement les femmes dans la planification de l'aide, et les traitent inégalement lors de la distribution. Des données qualitatives ont indiqué au $M$ alawi que les ménages de réfugiés mozambicains dirigés par desfemmes sont plus à risque queles autres par rapport à la santé et à la nutrition ${ }^{65}$.

Bien que les services médicaux dans les camps soient essentiels, les femmes n'y font pas nécessai rement appel. La communication avec le personnel majoritairement masculin constitue une contrainte majeure. Certaines femmes refusent de participer au dépistage de maladies contagieuses par peur du rejet familial et des conséquences sur leurs perspectives de mariage ${ }^{66}$.
La santé reproductive

En 1994, la santé reproductive a été reconnue comme un droit humain fondamental à la Conférence internationale sur la population et le développement au Caire. Afin de diminuer les taux de mortalité maternelle et infantile, conjointement avec d'autres agences, le HCR a défini un M inimum Initial Service Package (MISP) pour les situations d'urgence ${ }^{67}$. Les programmes de santé reproductive offrent des services de planification familiale (incluant les questions relatives à l'avortement) et des consultations prénatales et postnatales. Ils visent aussi à prévenir et à traiter lesmaladies sexuellement transmissibles, à prévenir et à gérer la violence sexuelle, et comportent des activités de sensibilisation aux pratiques traditionnelles néfastes.

LeH CR aégalement produitun manuel depratiquepour lepersonnel deterrain ${ }^{68}$. Cependant, en raison de contraintes budgétaires, la santé reproductive est rarement une priorité dans les camps de réfugiés. Les services négligent les adolescentes en visant prioritairement les femmes adultes ${ }^{69}$. Les réfugiées sont parfois les initiatrices de ces services; elles en ont ainsi catalysé l'introduction à Lumasi au Rwanda ${ }^{70}$. Dans la corne de l'Afrique, le HCR organise des activités de sensibilisation pour éliminer les croyances et pratiques nuisibles à la santé des femmes ${ }^{71}$. Elles visent à éliminer l'excision, le jeûne après l'accouchement et le mariage des fillettes. En effet, le mariage des adolescentes dès leur première menstruation entraîne des naissances prématurées $^{72}$. On tente aussi de démontrer aux hommes atteints du SIDA que contrairement à ce que prêchent certains guérisseurs traditionnels, unerelation sexuelleavec une jeune fille ne constitue pas un remède à la maladie ${ }^{73}$.

\section{La vulnérabilité des femmes et des filles réfugiées}

La vulnérabilité des femmes a été reconnue dans plusieurs résolutions internationales depuis le rapport du HCR à la World Conference of the U nited N ation's Decade for W omen $(1980)^{74}$. Ce thème est devenu prioritaire dans la littérature scientifique, qui traite les femmes en victimes plutôt que d'analyser le processus de transformation de leur vie $\mathrm{e}^{75}$. II faudrait étudier la discrimination et l'agression qui les frappent en raison de leur genre au lieu de mettre ainsi l'accent sur leur vulnérabilitép ${ }^{\text {. }}$.

Les violations des droits humains, la violence et la persécution basées sur le genre augmentent durant les guerres et les conflits armés. Elles constituent des tactiques de guerreet de terrorisme ${ }^{77}$. Alors que les hommes risquent de se faire battre ou tuer ${ }^{78}$, les femmes sont exposées à la violence sexuelle. C'est pourquoi le Sphere Project (1998), qui propose une charte humanitaire et des standards à respecter dans la gestion des désastres, insiste sur la prévention de la violence basée sur le genre et l'exploitation 
sexuelle $^{79}$. Les idéologies de genre existant en temps de paix auraient un impact sur les pratiques de genre durant les guerres ${ }^{80}$. Cette violenceliée au genrefait partie des processus globaux reliés aux relations de genreau sein du ménage et aux idéologies du chez-soi (home). L'identité de genre et par conséquent les ressources disponibles pour les réfugiées sont directement reliées à la manière dont le chez-soi et le ménage les ont historiquement définies en tant que femmes. Le ménage est vu ici comme une organisation sociale et économique qui supporte la survie de ses membres dispersés dans la diaspora à travers le monde. Le concept de chez-soi est un complexe d'idées qui se réfèrent à la sphère domestique, à la communauté et à la nation d'origine. Le corps de la femmedeviendrait ainsi la cible de la violence et le symbole de la nation violée. Le viol et la prostitution sont les deux formes de violence sexuelle les plus répandues. Le HCR a ainsi défini la violence sexuelle en 1987 :

[s] exual violence against women and girls usually occurs in situations in which women are forced into a relationship, a contact or an act in which they have no independent right to decide how they wish to behave with men. A feature of these situations is that women are restricted in their freedom and independence. Sexual violence takes on a variety of forms: assault, rape, sexual abuse of children, maltreatment of children, forcing women into prostitution or to pose for pornographic purposes. Violence may occur both in private and in public situations. A feature of all expressions of sexual violenceis thepsychological and/or physical violation of women's or girls' integrity ${ }^{81}$.

\section{La violence sexuelle}

En 1993, le Comitéexécutif du H CR a adoptéunerésolution recommandant le développement de mesures sur la protection des réfugiés et la violence sexuelle ${ }^{82}$ (UN HCR 1993 cité dans UN HCR 1995). En sebasant sur les recommandations de son personnel de terrain, le HCR a développé (1995) des directives administratives, médicales, psychologiques et légales afin de prévenir et de traiter les cas de violence sexuelle dans le contexte des camps ${ }^{83}$. Il a ainsi introduit des services d'aide psychosociale et médicale pour assister les femmes abusées physiquement ${ }^{84}$. II est toutefois déplorable que l'accent soit mis sur les services curatifs plutôt que sur des mesures préventives ${ }^{85}$.

Paradoxalement, alors qu'elles se réfugient pour fuir la persécution et la violence, les femmes augmentent le risque de violence sexuelle durant la fuite et l'exil ${ }^{86}$. La violence sexuelle engendre une souffrance immédiate et comporte des conséquences à long terme, telles que des traumatismes psychologiques, des grossesses non désirées, des maladies sexuellement transmissibles et le rejet familial.
Ainsi, des Éthiopiennes et des Érythréennes ont été torturées sexuellement en raison d'actions politiques par des membres de leur famille ${ }^{87}$. D'autres femmes sont agressées sexuellement par des individus qui utilisent leur position depouvoir dans les zones deconflits, aux frontières et dans les camps. L'exil les éloigne de leurs familles et de leurs protecteurs communautaires traditionnels ${ }^{88}$. Les rôles culturels stéréotypés et inégalitaires associés au genre, basés sur l'hypothèse que la femme est un être inférieur et dominé, perpétuent la violence sexuelle et domestique dans les camps ${ }^{89}$. Des membres de leur propre communauté profitent du démantèlement des structures et des valeurs sociales pour commettre de telles exactions ${ }^{90}$. Des femmes sont abusées par un membre du ménage ou par leur employeur, et durant les activités d'assistance humanitai re ${ }^{91}$. De même, les mineures non accompagnées habitant dans la famille élargie ou chez des non-parents sont parfois agressées sexuellement par les hommes du ménage ${ }^{92}$.

Les réfugiées soudanaises en Ouganda étaient violées al ors qu'elles tentaient d'assurer la survie de leur famille en cherchant de la nourriture ou en cultivant la terre ${ }^{93}$. Alors qu'elles avaient besoin de soins immédiats, les centres de santé avai ent été pillés ou manquaient de personnel qualifié. Les femmes violées avai ent honte et hésitai ent à dénoncer cet acte par peur d'être rejetées par la communauté ou abandonnées par leur mari. D es filles devenues les esclaves sexuelles des militaires par la force étaient rejetées lors de leur retour dans la communauté.

Les sévices sexuels commis contre les filles et les femmes tchadiennes en raison de la guerre civile sont bien documentés et démontrent que les forces gouvernementales et les rebelles y ont participé ${ }^{94}$. Elles sont violées par les militaires qui les soupçonnent de cacher les rebelles, et par ces derniersqui les accusent de les dénoncer au gouvernement.

Au Rwanda, des violences propres au contexte d'un conflit armé généralisé ont échappé au contrôle social pour s'étendre à presque toutes les femmes sans défense ${ }^{95}$. La cruauté physique s'est manifestée à travers le viol, la mutilation des organes associés à la reproduction, la servitude et le mariage forcé. Des hommes ont profité de la situation pour épouser les femmesles plus convoitées et leur imposer des conditions matrimonial es qu'elles auraient refusées en temps de paix.

\section{La prostitution}

La prostitution devient parfois une stratégie de survie de la part des femmes pour répondre à leurs besoins et à ceux de leurs dépendants. Leur mari lesforce parfois à se prostituer, et elles sont exploitées par les réfugiés entremetteurs, les clients et la police. C'est surtout le manque d'occasions d'emploi qui amène les chefs de ménage à se prostituer à 
l'intérieur ou à l'extérieur des camps96. L'existence du phénomène, bien que marginal, ternit la réputation de l'ensemble des réfugiées et provoque des abus physiques et psychologiques. Ainsi, les autorités et le public soudanais sont convaincus que la prostitution est très répandue chez les réfugiées éthiopiennes, alors que la plupart exercent une autre activité pour gagner leur vie. Celles qui ont recours à la prostitution ne le font que ponctuellement, quand elles n'ont pas de solutions de rechange, que les salaires sont faibles et le coût de la vie trop élevé. La prostitution met en péril leur sécuritéen augmentant leur vulnérabilitéau SIDA et aux grossesses non désirées. De plus, elle bouleverse le système de valeurs transmis aux jeunes générations. Ainsi, au Libéria, chez les personnes déplacées, l'activité sexuelle est devenue une stratégie de survie symboliquement courageuse, et elle constitue, avec le viol, un rite de passage vers I'âge adulte. Beaucoup de ces jeunes manquent d'encadrement et d'un système de valeur guidant leurs décisions et comportements sexuels $s^{97}$.

\section{Conclusion}

Cebilan des rapports degenre dans les situations de détresse a révélé les multiples facettes des inégalités engendrées par la migration forcée. Cependant, l'absencedecadrethéorique global limite la compréhension de l'effet du genre dans l'expérience du refuge. Un cadre analytique devrait être développé sur la base des nombreuses stratégies de survie socio-démographiques des individus, des ménages et des communautés. Comme les conséquences de la migration forcée sont vécues plus intensément à l'échelle du ménage, ce dernier constitue un objet d'étude important pour comprendrel'adaptation des réfugiés à leur nouvelle situation ${ }^{98}$. II serait certainement utile de considérer l'ensemble du processus de refuge dans le cadre d'une étude biographique visant la crise vécue dans le pays d'origine, la fuite, l'intégration dans le pays d'asile, la réinstallation dans un tiers pays et le rapatriement. De telles recherches devraient comparer la situation de réfugié dans le camp à celui dans les populations d'accueil. La situation des personnes déplacées pourrait être comparée à celle des réfugiés ayant traversé une frontièreinternationale. Cependant, un préalableà ces pistes de recherche est d'améliorer les méthodes de collecte de données auprès deces groupes vulnérables de la population. Ces informations permettraient certes d'améliorer l'aide humanitaire offerte aux réfugiés.

\section{Notes}

1. Indra Doreen, "Not a 'Room of One's Own' », dans Indra Doreen, Engendering Forced M igration. Theory and Practice (New York, Oxford: Berghahn Books, 1999), 1-22.
2. Heaven Crawley, «Gender, persecution and the concept of politics in the asylum determination process », Forced M igration Review 9 (December 2000) : 17-20.

3. Barbara E. Harrell-Bond, 1987, "Refugee Issues », dans P. Reason et]. Rowan, éd., H uman Inquiry. A SourceBook of $\mathrm{N}$ ew Paradigm Research (Chicester: John Wiley, 1987), cité dans M arie Indra Doreen, « Ethnic Human Rights and Feminist Theory: Gender Implications for Refugee Studies and Practice», Journal of Refugee Studies 2, no. 2 (1989) : 221- 42.

4. Helene M oussa, Storm \& Sanctuary. The J ourney of Ethiopian and Eritrean Women Refugees (Dundas, Ontario: Artemis Enterprises, 1993).

5. Doreen, «Ethnic Human Rights and Feminist Theory... ».

6. Ibid.

7. UNHCR, UNHCR Global Report 1999 (Genève: UNHCR, 2000).

8. UNHCR, A Framework for People-Oriented Planning in Refugee Situations T aking A ccount of W omen, $M$ en and Children. A Practical Tool for Refugee Workers. Mary B. Anderson, Ann Brazeau et Catherine Overholt (Genève: UNHCR, 1992).

9. Ibid.

10. UNH CR, «Executive Committee Conclusion N o. 73 (XLIV) (1993) on Refugee Protection and Sexual Violence », dans UNHCR, Sexual Violence against Refugees. Guidelines on Prevention and Response (Genève: UNHCR, 1995).

11. Ibid.

12. Ruth Marshall, «Refugees, feminine plural », Refugees 100 (1995) : 3-9.

13. Patrick M atlou, «Upsetting the Cart: Forced M igration and Gender Issues, the African Experience», dans Doreen, Engendering Forced M igration, 128-45.

14. Srilakshmi Gururaja, «Gender dimensions of displacement », Forced M igration Review 9 (December 2000) : 13-16.

15. Ibid.

16. Ainsi, selon la Convention (1951) et le Protocole des $\mathrm{N}$ ations Unies (1967) relatifs au statut des réfugiés, le terme réfugié s'applique à toute personne qui, craignant avec raison d'être persécutée du fait de sa race, de sa religion, de sa nationalité, de son appartenance à un certain groupe social ou de ses opinions politiques, se trouve en dehors du pays dont ellea la nationalité et qui ne peut ou, du fait de cette crainte, ne veut se réclamer de la protection de ce pays; ou qui, si elle n'a pas denationalité et se trouveà l'extérieur du pays où elle avait sa résidence habituelle à la suite de tels événements, ne peut ou, en raison de laditecrainte, neveut y retourner ( $N$ ations U nies, 1983). L'Organisation de I'U nité Africaine (OUA) a élargi cette définition en 1969 afin de l'adapter au contexte africain. Selon la Convention de l'OUA, « le terme réfugié s'applique également à toutepersonnequi, du fait d'uneagression, d'une occupation extérieure, d'une domination étrangère ou d'événements troublant gravement l'ordre public dans une partie ou la totalité de son pays d'origine ou du pays dont elle a la nationalité, est obligée de quitter sa résidence habituelle pour chercher refuge dansun autreendroit àl'extérieur deson 
pays d'origine ou du pays dont elle a la nationalité » (OUA, 1969).

17. M oussa, Storm \& Sanctuary. Crawley, «Gender, persecution and the concept of politics... ».

18. Crawley, «Gender, persecution and the concept of politics... ».

19. Nasreen $M$ ahmud, "Crimes Against H onour: Women in International Refugee Law », Journal of Refugee Studies 9, no. 4 (1996) : 367-82. Crawley, «Gender, persecution and the concept of politics... ».

20. Khadija Elmadmad, "M uslim Refugee Women », dans Doreen, Engendering Forced M igration. Theory and Practice, 261-71.

21. M oussa, Storm \& Sanctuary.

22. Johnson, 1989, cité dans Girma Kebbede, «The Agonies of Displacement: Ethiopian Women Refugees in Khartoum, Sudan », Geojournal 23, no. 2 (1991) : 99-106.

23. Nathalia Berkowitz, "Gender guidelines for the UK », Forced M igration Review 9 (December 2000) : 21.

24. UNHCR, UNHCR Global Report 1999 (2000).

25. UNHCR, UNHCR Issues - Women (2000) en ligne: $<$ <ttp://www.unhcr.ch/issues/women/women.htm>.

26. Patricia Daley, «Gender, Displacement and Social Reproduction: Settling Burundi Refugees in W estern Tanzania », Journal of Refugee Studies 4, no. 3 (1991) : 248-66.

27. M arleen Boelaert, Fabienne Vautier, Tine Dusauchoit, W im Van Damme et M onique Van Dormael, «The Relevance of Gendered Approaches to Refugee Health. A Case Study in H agadera, Kenya », dans Doreen, Engendering Forced M igration. Theory and Practice, 165-76.

28. Barbara E. Harrell-Bond, Imposing Aid. Emergency Assistance to Refugees (Oxford : University Press, 1986).

29. Kebbede, "The Agonies of Displacement... ».

30. M ekuria Bulcha, Flight and Integration. Causes of $M$ ass Exodus from Ethiopia and Problems of Integration in the Sudan (U ppsala : Scandinavian Institute of African Studies, 1988).

31. UNHCR, Unit Plan and lessons plans for ages 15-18 in Civic Education: 'Refugee Women' (2000) en ligne: <http://www. unhcr.ch/teach/tchcived/15-181p.htm>.

32. UNHCR, The State of The W orld's Refugees in search of solutions. Oxford University Press (1995) en ligne: <http:// www.unhcr.ch/ refworld/pub/state/95/box2_1.htm>.

33. UNHCR, «Women in war-torn societies», dans UNHCR, The State of the World's Refugees -A Humanitarian Agenda (1997) en ligne: <http://www.unhcr.ch/refworld/pub/state/ 97/box4_3.htm>.

34. Boelaert et al, «The Relevance of Gendered Approaches to Refugee $\mathrm{H}$ ealth... ».

35. Daley, «Gender, Displacement and Social Reproduction... ».

36. Lucia Ann M cspadden et Helene M oussa, «I Have a Name: The Gender Dynamics in Asylum and in Resettlement of Ethiopian and Eritrean Refugees in N orth America », Journal of Refugee Studies 6, no. 3 (1993) : 203-25.

37. Simon Turner, «Vindicating masculinity: the fate of promoting gender equality », Forced M igration Review 9 (December 2000) : 8-9.
38. Lina Payne, «Food Shortages and gender relations in Ikafe settlement, Uganda », Gender and Development 6, no. 1 (M arch 1998) : 30-36.

39. Callaway, 1985, citédans Kebbede, «TheAgonies of Displacement... ».

40. Daley, «Gender, Displacement and Social Reproduction... ».

41. Doreen, «Ethnic Human Rights and Feminist Theory... ».

42. H arrell-Bond, Imposing Aid. Emergency Assistance to Refugees.

43. John Sorenson, «An Overview: Refugees and Development», dans Adelman Howard et John Sorenson, African Refugees. Development Aid and Repatriation (Boulder et Londres: Westview Press, 1994), 175-90.

44. Christiane Berthiaume, "Do we really care? », Refugees 100 (1995) : 12-13.

45. Wallace Tina, «Taking the lion by the whiskers: building on the strengths of refugee women », dans Wallace Tina et Candida M arch, Changing Perceptions. Writings on Gender and Development (Oxford: Oxfam, 1991), 60-67.

46. UNHCR, "Refugee Women », dans UNHCR, 1999 Global Appeal and Programme Overview (1999) en ligne: <http://www.unhcr.ch/fdrs/ga99/women.htm>.

47. Ibid.

48. Harrell-Bond, Imposing Aid. Emergency Assistance to Refugees.

49. M oussa, Storm \& Sanctuary.

50. Daley, «Gender, Displacement and Social Reproduction... ».

51. Ibid.

52. Payne, «Food Shortages and gender relations in Ikafe settlement, U ganda ».

53. M adi Passang et N oelle N odjal, The flight, exile and return of chadian refugees. A case study with a special focus on women. A synthesis of research conducted by M adi Passang, N oelleN odjal and the research team of the Chadian National Institute of Social Sciences. Prepared by Carol Watson for the United Nations Research Institute for Social Development (1996).

54. Payne, «Food Shortages and gender relations in Ikafe settlement, U ganda ».

55. Nasreen $M$ ahmud, «Crimes Against H onour: Women in International Refugee Law ».

56. Tina, "Taking the lion by the whiskers: building on the strengths of refugee women ».

57. M arshall, «Refugees, feminine plural ».

58. Doreen, «Ethnic Human Rights and Feminist Theory... ».

59. Tina, "Taking the lion by the whiskers: building on the strengths of refugee women 》.

60. Marshall, «Refugees, feminine plural ».

61. UNHCR, UNHCR Issues - Women (2000) en ligne: $<h t t p: / / w w w . u n h c r . c h / i s s u e s /$ women/women.htm>.

62. Boelaert et al, «The Relevance of Gendered Approaches to Refugee Health... ».

63. UNHCR, H ealth Careand the Refugee Family (1995) en ligne: <http://www.unhcr.ch/issues/women/rm09510.htm.

64. Boelaert et al, «The Relevance of Gendered Approaches to Refugee $\mathrm{H}$ ealth... ». 
65. Alastair Ager, Wendy Ager et Lynellyn Long, «The Differential Experience of M ozambican Refugee Women and M en », Journal of Refugee Studies 8, no. 3 (1995) : 265- 87.

66. Boelaert et al, «The Relevance of Gendered Approaches to Refugee Health... ».

67. M édecins sans frontières, Refugee $\mathrm{H}$ ealth. An approach to emergency situations (Londres: M acmillan, 1997).

68. M arshall, «Refugees, feminine plural ».

69. Nanette Ecker, «Where There is No Village: Teaching About Sexuality in Crisis Situations », Siecus Report 26, no. 5 (June/July 1998) : 7-10.

70. Heather Courtney, Spacing children, preventing AIDS (1995) en ligne: <http://www.unhcr.ch/issues/women/ rm10005. htm>.

71. UNHCR, UNHCR Global Report 1999 (2000).

72. Ecker, «Where ThereisNo Village: TeachingA bout Sexuality in Crisis Situations ».

73. Ibid.

74. M oussa, Storm \& Sanctuary.

75. Daley, «Gender, Displacement and Social Reproduction... ».

76. M oussa, Storm \& Sanctuary.

77. UNHCR, «Refugee Women », dans UNHCR, 1999 Global Appeal and Programme Overview (1999) en ligne: <http://www.unhcr. ch/fdrs/ga99/women.htm>.

78. Payne, «Food Shortages and gender relations in Ikafe settlement, U ganda ». M cspadden et M oussa, «I H avea Name... ».

79. Gururaja, «Gender dimensions of displacement ».

80. Wenona Giles, «Gendered Violence in War », dans Doreen, Engendering Forced M igration.

81. UNHCR, 1991, citédans M ahmud, «Crimes Against H onour: Women in International Refugee Law »: 368.

82. UNHCR, «Executive Committee Conclusion No. 73 (XLIV) (1993) on Refugee Protection and Sexual Violence».

83. UNHCR, UNHCR Issues - Women (2000).

84. UNHCR, "Refugee Women », dans UNHCR, 1999 Global Appeal and Programme Overview (1999) en ligne: $<$ <ttp://www.unhcr. ch/fdrs/ga99/women.htm>.

85. Indra Doreen, «Interview with Barbara Harrell-Bond », dans Doreen, Engendering Forced M igration.

86. UNHCR, TheStateof theW orld'sR efugeesin search of solutions. Oxford U niversity Press (1995) en ligne: <http://www.unhcr. ch/ refworld/pub/state/95/ box2_1.htm>.

87. M oussa, Storm \& Sanctuary.
88. Mahmud, «Crimes Against Honour: Women in International Refugee Law ». M cspadden et M oussa, «I H ave a N ame... ».

89. Ecker, «WhereThere is No Village: Teaching About Sexuality in Crisis Situations».

90. UNHCR, TheState of theW orld's Refugeesin search of solutions.

91. Kebbede, «The Agonies of Displacement: Ethiopian Women Refugees in Khartoum, Sudan ».

92. Ecker, «Where There is No Village: Teaching About Sexuality in Crisis Situations».

93. Payne, «Food Shortages and gender relations in Ikafe settlement, U ganda ».

94. Women's Commission of the H uman Rights League of Chad $\&$ the Editors, «Women Denounce Their Treatment in Chad », dans Meredeth Turshen et Clotilde Twagiramariya, What Women Do in Wartime (Londres, New York: Zed Books Ltd, 1998), 118-28.

95. Édith M ukakayumba, «Rwanda : la violence faite aux femmes en contexte de conflit armé général isé », Recherches féministes 8, no. 1 (1995) : 145-54.

96. Tina, « Taking the lion by the whiskers: building on the strengths of refugee women ».

97. Ecker, «WhereThere is N o Village: Teaching About Sexuality in Crisis Situations ».

98. Atsuko Matsuoka et John Sorenson, «Eritrean Canadian RefugeeH ouseholds As Sites of Gender Renegotiation », dans Doreen, Engendering Forced M igration, 218-41.

L'auteure est titulaire d'un PhD du Département de Démographie de l'U niversité de M ontréal. Ellea alors étudié l'effet de la migration de refuge sur la nuptialité tchadienne. Elley a aussi réaliséune maîtrise en démographie portant sur I'universalisation de l'immigration internationale au Canada. Elle est présentement chargée de programmes pour l'Afrique del'O uest dansl'organismenon gouvernemental World Vision Canada. À ce titre, elle est responsable du design, du suivi et del'évaluation de programmesmultisectoriels dansles pays suivants : Ghana, M ali, M auritanie, Niger, Sénégal, Sierra Leone et Tchad. Elle est intéressée par les questions relatives aux réfugiés et aux personnes déplacées, plus particulièrement à la problématique des femmes et des enfants dans le contexte des conflits armés. 